\title{
Quelques livres du canon littéraire roumain- face au pouvoir politique
}

\section{Les conflits / les confins canoniques - \\ L'ethos national ou l'esprit universel}

Il est communément accepté le fait que le canon littéraire est agonistique, parce qu'il fait des hiérarchies, il est conflictuel parce qu'il met en compétition le nouveau avec la tradition littéraire. Le canon est aussi un produit culturel transnational, syncrétique et cosmopolite, exprimant les rapports entre l'ordre universelle et l'ordre national, entre les normes académique et les tendances expérimentales, entre les valeurs, les modes de dictions, les artistes des grands littérature et les modèles, les valeurs, les écrivains des petites littérature nationales.

Quand Milan Kundera soutient dans son essai sur l'histoire du roman, Le Rideau (2005), l'idée que les oeuvres essentielles de la littérature européenne doivent être comprises dans le concept paradigmatique de «Weltliteratur $\gg$, en fait, il nous pose la question fondamentale sur le destin des oeuvres des petites cultures. En même temps, l'esprit critique de synthèse cosmopolite de Kundera met en lumière les deux aspects du provincialisme : celui des petits et celui des grands ; c'est-à-dire, les complexes des marginaux, des cultures mineures, qui tombent dans le nationalisme, dans les stéréotypes identitaires (qui doivent justifier leur force et leur spécificité par le «terrorisme du petit contexte » qui réduit le pluralisme de l'interprétation et la signification de l'œuvre au rôle déterminé seulement pour l'usage du contexte national), et aussi, le provincialisme des grandes cultures, ou des cultures majeures, qui (s)ont instauré(es dans) l'indifférence et le culte de l'oubli de la valeur esthétique.

En ce qui concerne l'homme de l'est, Kundera trace des lignes très sûres pour dessiner une mappe qu'il peut nous sembler étrange si nous la regardons de l'ouest, c'est le territoire spirituel des petites nations de l'ancien Empire de Habsbourg et en fait c'est le territoire polycentrique de l'Europe Centrale, malgré son « caractère vague et approximatif ». Dans sa socialité des confins indéterminés ou relativement, arbitrairement décelées par des traités politiques, par les contrats officiels, ce territoire devient un topos imaginaire, le 
ILIE

cadre de la pluralité culturelle, du « maximum de diversité dans le minimum de l'espace $\gg$ (Kundera 2008: 37-61).

Donc, les tensions canoniques européennes ne doivent pas être réduites seulement à la compétition des grandes littératures, aujourd'hui, elles doivent être comprises dans le dynamisme culturel qui engendre à la fois les énergies nationales et les énergies universels, l'ethos national et l'esprit de synthèse transnational et transhistorique, la généricité et la spécificité, l'universalisme et l'exotisme.

L'état de conflit culturel est aboli dans les accords canoniques, mais ces dernières sont possibles par le dialogue entre les cultures régionales, par le désir de la communication égalitaire, démocratique, ou la tolérance n'est pas une attitude artificielle, soutenue par les jeux de l'emphase politique globalisante, mais une attitude soutenue par la voie réelle de la diversité/ de la différence. Ce n'est que dans ce sens on peut parler du canon européen comme d'un lieu de la mémoire (Steiner 2006). La connaissance et l'exploration de cet espace sont possibles par l'abolition des stéréotypes sociaux, religieux et culturels, par la chance que la musique (comme langage universel) et la littérature (comme langage particulier, insolite et divers) offre à l'homme pour qu'il puisse franchir les barrières linguistiques, ethniques.

La culture européenne moderne et contemporaine s'impose, donc, par la négociation des positions dans le champ des productions littéraires, dans le cadre et le circuit des valeurs par les mécanismes qui survolent les blocages, les limites nationales, géopolitiques. Les voyages, les traductions, le changement de mémoire culturelle et le modèle de pardon, les dialogues de l'autre avec l'autrui dans l'exile ou dans des autres situations tragiques (comme celle de la déportation, de l'extermination ou de l'univers carré des régimes totalitaires) sont les manières sensibles, subjectives et concrètes de la communauté de l'esprit européen, comme l'a souligné George Steiner (2006) et comme Paul Ricœur l'a illustré, lui-aussi (2004).

La prose produite sous les dictatures est un exemple révélateur pour montrer la résistance, la force anti-idéologique qui a aboli les barrières dogmatiques, en s'assumant la dissidence de l'esprit libre, l'exclusion volontaire et la position anti-canonique, anti-officielle. L'intensité fébrile de l'action créative et critique des écrivains de la résistance anti-communiste roumaine, hongroise, slovaque, ou baltique se mesure dans les cadres désignés par Milan Kundera par sa pléiade centrale européenne. Car l'expérimentalisme, l'édifice irrégulier de la narration, l'intensité anti-lyrique et la fascination pour l'ambiguïté corrosive de la phrase ont origines dans «la solidarité » singulière 
de Kafka, Musil, Gombrowicz et Broch. Avec Milan Kundera, on peut dire que « les aspects jusqu'alors inconnus de l'existence que ce roman a su éclairer ; la nouveauté de la forme qu'il a su trouver $\gg(2008: 56)$ sont les traits spécifiques de la prose de la fin de XX-ème siècle, pendant que les écrivains sont devenus des poètes de la forme, en élaborant une esthétique du roman face à la morale et à la politique du jour. Une esthétique non - idéaliste, contre-discoursive, accompagnée par la pratique de l'expérimentalisme formel, structurel, ayant comme but seulement la valeur lancée dans la querelles des cultures centrales/grandes/ majeures et des cultures nationales/ régionales/ petites qui cherchent la chance d'être reconnues dans le grand contexte universel.

Leur chance est jouée, comme l'a montré Kundera, justement dans les biais de leur excentricité linguistique, dans le paysage musical, expressif et imaginatif de leur individualité irréductible, qui a caché, qui a préservé le patrimoine culturel de l'authenticité et de la différence, de la noblesse de l'esprit libre. Ce patrimoine de la mémoire culturelle n'est pas le dépositaire des valeurs d'une manière artificielles, il est le paradigme catalytique qui s'exerce dans les termes de l'exemplarité et de l'immortalité des chefs-d'œuvre. Et chaque chef-d'œuvre représente une ou plusieurs questions permanentes dans le renouvellement des fondements de l'humanité.

\section{Formes et codes esthétique face au pouvoir politique}

Le contexte littéraire roumain de la fin du régime communiste remet en question les formules, les codes artistiques, les stratégies narratives, car la pression idéologique a imposé une direction spéciale, des lignes d'évasion, des modalités qui ont exprimé en fait la subversion des canons et des directives officiels. La force et aussi la résistance anti-canonique de cette espèce de subversion consistent dans les tactiques expérimentales qui ont leur fonction de diversion par les discours ironiques, ludiques, paralogiques, allégoriques. Tel que le souligne Monica Spiridon, dans une terminologie spécifique à la combativité forcée d'être réduite au silence, «Au-delà des campagnes expérimentales, on jouait une carte essentielle pour le destin de la littérature roumaine: d'être en synchronie avec l'esprit du temps, la conscience de l'appartenance européenne, l'orientation pro-occidentale de l'intellectuel roumain». (1998: 9-19).

Le champ de production littéraire (Bourdieu 1992) de cette période peut être configuré dans un carré qui pose des écrivains dans de tacites positions conflictuelles. Certains pratiquent la littérature convergente aux prescriptions 
du Parti unique, une littérature servile et conformiste aux attitudes imposées par le système, une littérature des bards de la cour; ce sont les écrivains agréés, tels Titus Popovici, Dumitru Popescu, Corneliu Vadim Tudor, Zaharia Stancu, Mihai Beniuc, Adrian Păunescu. À cette littérature opportuniste s'oppose la littérature subversive, désignée ainsi par Ion Simuț («Care patru literaturi ?» 1993), une littérature très riche, illustrée par des noms comme Marin Preda, Augustin Buzura, Ana Blandiana, Mircea Dinescu, Nicolae Steinhardt, Marin Sorescu, Ileana Mălăncioiu, etc. D’autre part, il y a une littérature dissidente, qui radicalise les efforts contre-idéologiques et esthétiques des écrivains en opposition avec le régime, en affirmant l'esprit critique et la lucidité, la pratique authentique de la conscience publique, la tension de la liberté d'expression morale, définie comme «la résistance par la culture». Les représentants de cette option sont Paul Goma (qui a d'abord publié ses livres à l'étranger, par exemple Ostinato, refusé en Roumanie et publié en 1971 aux Editions Gallimard et Suhrkamp en français et en allemand, puis, en néerlandais, en 1974, finalement en roumain, 1992; Gherla, en français 1976, en suédois 1978, en roumain, 1990, or La couleur de l'arc-en-ciel en français 1979, néerlandais, 1980, finalement en roumain, en 1990), Ion Negoițescu, Dorin Tudoran, Dan Petrescu, Mircea Dinescu sont punis d'une manière drastique par la censure.

Ioana Macrea-Toma, qui fait un étude analytique sur la censure «institutionnalisée et incorporée», en illustrant les stratégies du régime des publications dans la Roumanie communiste, affirme que

La censure, définie par certains sociologues comme toute intervention d'une autorité gouvernementale destinée à empêcher ou à limiter la diffusion des livres ou des périodiques ou, plus largement, comme phénomène qui intervient entre les différents producteurs des discours pour régler un champ conflictuel de relations symboliques, fait partie, dans les régimes communistes, du champ de la production idéologique qui légifère la constitution d'une réalité unique et adéquate au dogme du Parti. (Macrea-Toma 2011)

Pour se sauver de la surveillance soutenue de la censure politique et du processus d'épuration de leurs textes, quelques - uns des écrivains roumains envoient leurs œuvres dans la diaspora, où la résistance culturelle est soutenue par les efforts de Monica Lovinescu et Virgil Ierunca.

Accepter que la subversion politique suppose une subversion cognitive, une conversion de la vision du monde et que, de ce point de vue, la théorie marxiste « a exercé un effet de théorie sans équivalent dans l'histoire » par l'ajustement 
Quelques Livres du Canon Littéraire Roumain - face au pouvoir politique

des pratiques institutionnalisées aux discours scientifiques, nous aide à mieux comprendre l'ampleur indéfinie du contrôle des informations et de la communication dans les pays socialistes. (Ib.)

En conséquence, la dernière catégorie qui joue dans la scène littéraire de la période 70-80 selon Ion Simuț, sont les écrivains qui ont pratiqué le discours apolitique, les belles-lettres-istes, les “évasionistes”, les réfugiés dans l'espace serein et pur de la Littérature (Maurice Blanchot), comme Nichita Stănescu, Leonid Dimov, Emil Brumaru, Petre Stoica, Ştefan Bănulescu, avec les utopies du langage, les fantasmes de l'onirisme, la « réservation des anges » comme topos imaginaire bachelardien qui assure la plénitude de l'individu dans la primauté des matières, des objets domestiques, des sensations nostalgiquement filtrées dans les poèmes de la sagesse, naïve ou corrompue, des jeux du langage, des jeux aux accents surréalistes, intimistes, etc.

Cela doit souligner l'existence d'une autre formule, fortement autoréflexive, ludique et ironique dans les écrits de l'école de Târgovişte (Radu Petrescu, Mircea Horia Simionescu, Tudor Țopa, Costache Olăreanu), de Şerban Foarță, de Mircea Cărtărescu, quand, après le hiatus proletcultiste, la vitalité productive de la prose expérimentale semble avoir un parcours normal, au-delà du réalisme socialiste et du militantisme qui a exploité toutes les formes de la propagande totalitaire.

Les expérimentes esthétiques sont devenus la stratégie pour assurer la légitimation dans la littérature contemporaine. Une possible influence se trouve dans l'expérimentalisme du Gruppo 63, I verri, dans la poétique du Nouveau Roman français, dans le mouvement Tel Quel, dans les néo-avantgardismes: par exemple, Gellu Naum, Nichita Stănescu, Mircea Ivănescu, Constantin Abăluță, Marin Sorescu, Virgil Mazilescu imposent «le livresque ostentatoire, l'abstraction de la lyrique et l'irréalité de la parole, la déstructuration radicale de la cohérence du réel $\gg$. Ceux-ci deviennent les effets artistiques d'une crise de la représentation, qui entretiennent la fièvre antimimétique, du refus de l'histoire.

La littérature des années 70-80 acquiert une fonction critique marquée par la re-écriture de l'histoire, issue d'un désir de refondation du monde et du langage, expérience qui se traduit par des notes des aventures quotidiennes dans des topoï anticulturels, naturels, dépaysés, marginaux et ambigus comme manières d'existence, tels que les marécages, les périphéries, les espaces de passage antisociaux spécifiques aux pérégrinations des personnages de Zenobia de Gellu Naum ou de Zacharias Lichter, le personnage du roman de Matei 
Călinescu. Le caractère transgressif de ces romans consiste dans les modalités de relativisation de la narration, dans le camouflage lyrico-essaystique de certaines prises de position des intellectuels qui ont choisi ce type de discours romanesque pour répondre de biais aux graves écarts de la normalité critique et de la responsabilité concernant la liberté de pensée et de communication publique et privée qu'impose la littérature comme forme de la conscience morale générale.

Dans ce contexte, dans les années '80, Mircea Nedelciu exprimait de façon très aiguë, prégnante et articulée sa position d'écrivain obligé de former des consciences réflexives, autonomes et authentiques. Voilà son credo artistique enregistré par la revue littéraire Ateneu:

Tout d'abord, l'écrivain a une responsabilité quant à son peuple, dans la présente situation. Je parle donc du présent où il écrit. En même temps, il a une responsabilité quant à la littérature - et je parle ici de toutes les époques. Cette double responsabilité de l'écrivain en devient finalement une quant à l'humanité en général, et je considère qu'écrire signifie, au fait, faire une oeuvre d'anthropogénie, intervenir dans la constitution de l'homme; à la question si la littérature peut faire quelque chose pour l'homme, un écrivain chinois a très beau répondu: Non, la littérature est celle qui justement fait l'homme. («Scriitorul tânăr şi actualitatea», débat avec Constantin Vişan, apud Lefter 2010: 38-39).

Si le roman de l'obsédante décennie avait produit les romans du grandiose et du sublime, qui officiaient le culte de la personnalité unique et légitimaient le pouvoir politique par les méta-narrations bien connues, cultivées par l'idéologie gauchiste (l'Histoire, Le Leader, le Progrès et le projet de l'Homme Nouveau), les formes de la nouvelle prose sont minimalistes, elles font éclater les prescriptions du réalisme, en inventant « des moyens ingénieux de neutralisation » (Spiridon 1998: 15). Le fragmentarisme des visions, le dévoilement voilé, en anamorphose, la réécriture ironique ou parodique qui relativise et hybride les modèles, l'esthétique de la discontinuité (Musat 1998: 24), de la recherche d'une forme narrative qui semble ne se coaguler que par l'intervention autoréflexive, de notation narcissiste, auctoriale qui concilie à peine l'extérieur de la réception à l'édifice esthétique, l'historicité à la transhistoire, sont des stratégies de fondation d'un autre discours culturel, et non seulement littéraire. La réponse qu'offre ce nouveau modèle narratif est non seulement expérimentale, mais existentielle, ne soulignant pas seulement la virtuosité de l'écriture de certains maîtres de notre prose postmoderne, mais surtout leur vision et leur option 
socio-morale. Les lettres, les réponses à différentes enquêtes littéraires, les interviews de Nedelciu, de Crăciun etc. dépassent le cadre intime, amical, arrivent à des problématisations culturelles graves, à des réponses plurielles et ancrées dans la dimension européenne des idées.

La réflexion sur le devoir de l'écriture souligne la haute conscience morale et le dévouement de l'auteur qui ne considère pas le lecteur comme un intrus, comme un passif récepteur ou comme un destinataire de la manipulation artistique, mais comme un partenaire de dialogue, qui l'attend et le prend amicalement dans le jeu de la communication symbolique:

Jusque récemment, ma position de travail supposait que l'action d'écrire est une sorte d'anthropogénie. Le but de l'écriture serait de transformer l'homme, les techniques et les méthodes d'implication du lecteur dans l'oeuvre et le contrôle des effets sur les consciences et l'affectivité. Je ne regrette point d'avoir "fouillé" dans cette direction. J'ai appris un tas de choses sur les fonctions de manipulation que peut avoir l'art et qui sont souvent exploitées par des forces extérieures à la littérature. J'ai pu aussi observer que l'art peut renforcer l'âme et le cerveau contre les manipulations de tout genre. Le dernier temps je crois de plus en plus fermement que la littérature est toutefois une recherche individuelle de la vérité sur l'homme, et que cette fonction "anthropogénique" est collatérale. Je veux dire que l'homme est un miracle de l'univers en tant que tel. Il sait à lui tout seul se former et résister à la manipulation. La littérature peut, tout au plus, stimuler l'autoconnaissance et c'est déjà grand-chose. (apud Lefer 2010: 39).

On peut remarquer que dans ses lignes fortement tracées l'écrivain expose la vérité que la pensée politique du contexte communiste ne pourrait pas l'accepter sans une modulation rhétorique, sans un maquillage idéologique, mais Nedelciu le pose d'une manière transparente et ouverte dans son discours en légitimant l'activité libre de l'esprit, malgré les réglementations de la censure. Il faut souligner le courage de l'affirmation dans le contexte de sa publication: la revue dans laquelle Nedelciu expose sa théorie est Scânteia tineretului. Suplimentul literar-artistic, revue affiliée aux orientations gauchistes, l'instrument médiatique du Parti communiste. Seul le titre de l'interview réalisée par Gabriela Hurezean détourne le sens individuel contenu par la vision de l'auteur vers le sens impersonnel, commun, de l'idéologie, vers l'horizon d'attente des censeurs: « Je ne crois pas à la solitude absolue de celui qui écrit », qui semble porter la signification convenable dans le contexte de l'engagement politique, dans les efforts de l'activisme collectif. Mais, les phrases 
de Nedelciu, tout en usant du langage officiel, sont exprimées de manière antiphrastique, étant en même temps transitives, ironiques et subversives.

Ainsi, autant dans la théorie que dans la prose, au canon officiel on apporte pour la première fois de façon programmatique, radicale et soutenue par "la science du texte", une alternative, des contre-langages et un contre-canon. Celui-ci n'est pas revendiqué, il n'est publiquement assumé et intégré que très tard, il reste plutôt underground, même lorsque ses représentants parviennent au champ culturel par le truchement des cénacles littéraires, par l'appui à la publication accordé par certaines revues estudiantines, Echinox, Dialog, România Literara etc. Carmen Musat observe que ce contre-canon, «nonofficiel, fortement culturel », a le mérite d'offrir «une image beaucoup plus cohérente de la littérature roumaine pendant le régime communiste, qui ne justifie pas l'idée d'une "Sibérie culturelle" le long de ces années et qui représente une vengeance de l'esthétique et de la liberté intérieure contre la nullité idéologisante de certaines pséudo-valeurs consacrées par la propagande $\gg($ 1998: 19-20).

Le refus du conformisme technique de la poésie et de la prose, dans une époque obsédée par l'idée du monumental dans la civilisation et la culture conduit à des déviances, à des écarts et des camouflages de ces tendances de rejet du dictat idéologique réalisé autant au niveau thématique, (la problématique romanesque officielle étant rejetée, ocultée), qu'au niveau de la philosophie et de la composition du roman, qui par des ingéniosités structurales, par une écriture sophistiquée, à discontinnuités narratives, avec la segmentation des perspectives de focalisation, avec des évasions de la «normalité » du présent ou des retours spectaculaires de nature métadiscursive, autoréférentielles, autodestructrices, font la preuve d'une réponse courageuse, pluriellement assumée, de désémantisation et resémantisation par la réécriture, en alternant avec la vision unique sur le monde, promue par l'idéologie officielle. Le canon esthétique est structuré par l'exercice des libertés expérimentales, de pratique d'une écriture qui relativise les modèles antérieurs, les décompose et les sape par l'ironie, même si parfois les thèmes et les personnages sont les mêmes (v. Nedelciu: Griguță, Fatache, les personnages de la prose Efectul de ecou controlat / L'effet d'écho controlé).

D'autres formules de discours: I. B. Lefter inventorie, à la suite du passage de la «révolution» prolétarienne à la «restauration» moderniste, des formes et des structures comme celles de l'avant-garde perpétuelle (Gellu Naum, Sebastian Reichmann, Valeriu Oişteanu, Dan Stanciu etc.), de «l'extrémisme» livresque (Vulpescu, Negoițescu, Doinaş, Adrian Popescu, Dinu Flămând, 
Tudor George, Teodor Pîcă, Şerban Foarță), de l'expérimentalisme morphologique (Şerban Foarță des années 80, Adrian Rogoz), des «proustianismes, joycéanismes, faulknérianismes nouvelle école» (Al.Ivasiuc, G.Bălăiță, Dumitru Radu Popescu), des échos du nouveau roman (Tepeneag, Goma, V. Andru); es premiers expérimentalismes: l'onirisme poétique, Dimov, la poésie du prosaisme biographique (Mircea Ivănescu, Petre Stoica, Florin Mugur, Dorin Tudoran), la poésie sensuelle à objets animés (Brumaru), la poésie surétagée (Radu Petrescu, M.H. Simionescu, C. Olăreanu, Petru Creția, Al George), les romans de l'essayisme énigmatique (Matei Călinescu, Viata şi opiniile lui Zacharias Lichter, 1968; Virgil Nemoianu, Simptome, 1969; Toma Pavel Fragmente despre cuvinte, 1968; Vintilă Ivănceanu, Până la dispariție, 1968; Nemaipomenitele pățanii ale lui Milorad de Bouteille, 1970), histrioniques, parodiques, commencent la comédie postmoderne, à goût amer, de subversion et répression de la liberté créatrice plénière.

\section{Au-delà de l'expérimentalisme, l'authenticité}

Bien qu'à l'époque ils fussent considérés comme des marginaux, liés au système de façon paradoxale par leur position contre-idéologique, les '80-istes ont exprimé avec prégnance la désillusion, le dégoût, leur opposition au manque de normalité de l'expression libre. Comme le note Andrei Bodiu, «en 1980, contre une réalité sociale cruelle, en dépit du manque de liberté, une nouvelle direction était née dans la littérature roumaine» (2000:15).

$\mathrm{Au}$ fait, ce qui a le plus affecté la présence de ces écrivains a été leur marginalisation, le refus de les publier ou de les accepter au champ des débats publics, le blocage de leur participation à la vie culturelle réelle, en dehors des enclaves estudiantines, littéraires. Il est à remarquer que ce ne fut pas forcément leur œuvre a avoir souffert des modifications intrinsèques de substance ou de vision, car les auteurs soit gardaient l'œuvre «dans le tiroir» pour d'autres époques, soit avaient déjà trouvé les stratégies esthétiques de salut de la suffocante pression qu'imposait la censure. La dure réalité autobiographique, le caractère de «sémi-légalité» du groupement et «le statuts social ambigu», les pressions, les ajournements éditoriaux (ib. 17-19), les contraintes esthétiques déterminent chez les poètes et prosateurs 80 -istes de nouvelles formules de résistance face aux thèmes du réalisme socialiste, exploitées par des codes ludiques, subversifs, intertextuels. Le refus ostentatoire de l'engagement et les tensions évidentes produites par le renoncement aux compromissions, fussentelles d'ordre esthétique, rendirent les valeurs de ces écrivains contre-canoni- 
ques, radicalement différentes par rapport à celles des contemporains des générations de création antérieures.

En outre, le contexte fit que tant la prose, que la poésie des 80 -istes définît une autre manière de solidarité, évidement humaine et intellectuelle, nonidéologique et non-partisane, fondée sur l'esprit d'opposition au système soutenue par l'amitié de véritables personnalités critiques (Nicolae Manolescu, Mircea Martin, Ion Pop, Ov. S. Crohmălniceanu), ainsi que par l'amitié, la solidarité et la sincérité des individualités créatrices elles-mêmes. Les jeux de l'esprit innovateur - agonal, qui construisaient un contrediscours littéraire risquant à démeurer conjoncturel, ont articulé de la façon la plus cohérente une nouvelle littérature, dont la personnalité devint autonome et essentiellement canonique pour le postmodernisme roumain. De l'expérimentalisme ostentatoire des pionniers, de la légitimation théorique extérieure et assumée en programme (par l'influence du textualisme français sur la prose et de la littérature anglo-américaine sur la poésie) on arrive nécessairement au nonconformisme existentiel, à la définition critique originale et à l'affirmation ontologique du désir d'authenticité.

Dans sa série d'études publiées posthume, Gheorghe Crăciun (2009) s'est proposé d'aborder d'une manière indirecte la problématique de l'authenticité, en analysant les écrits d'auteurs sélectionnés au-delà de leurs sujets, «dans leur pure motivation psychique et existentielle, en suivant l'origine de la pulsion d'écrire, sa motivation, l'authenticité et l'autodiscipline de cette pulsion, son administration jusqu'à l'achèvement dans l'oeuvre». Dans l'opération de cartographier la poétique de la prose de M. Nedelciu, dès son début, ce qui lui semble symptomatique dans l'écriture de son ami, est «le refus du corps intime, du corps subjectif comme alternative identitaire aux pressions $\mathrm{du}$ monde extérieur. Il ne s'agit pas d'un refus de facto, ni d'un déficit de potentiel somatographique de l'auteur, mais du saisissement du conflit irréductible entre le corps et le langage» (Crăciun 2009: 71). Sans la focalisation dominante sur le contexte historique, le critique souligne le caractère essentiel de la prose nedelcienne. Comme activité consciente, la littérature roumaine des années ' 80 a une fonction ambiguë, elle est transfigurée dans une sorte de pharmakon. Chaque pacte de l'écriture a le but de remédier à une crise, à une maléfique action de l'histoire, à une rupture, et donc son résultat devient ontologique, se constitue dans une thérapie par l'art. Mais aussi, comme pour le pharmakon, son effet peut être contraire, peut perpétuer le maléfice, la malédiction de l'époque, par des contre-langages, fréquemment «accéptés », tolérés par la censure. Donc, le langage de Nedelciu se constitue dans le message d'une 
Quelques Livres du Canon Littéraire Roumain - face au pouvoir politique

étrangeté ayant l'effet «d'écho contrôlé», car la transitivité cache l'ironie et le sens souterrain, la narrativité donne et, en même temps, oblitère la vision critique, le dévoilement éthique:

Dans les livres de M. Nedelciu, le corps individuel est saisi et décrit dans ses formes socialisées, d'action et de présence publique, mais dans ce cas aussi l'élément généalogique ou économique sont plus importants que les ressorts psycho-pulsionnels de l'existence. La suggestion de l'appartenance du corps individuel à un corps-masse, guidé par des stéréotypies fondamentales, à fortes insertions historiques, est elle-même présente. Les personnages de l'auteur se constituent en couples, groupes, familles, catégories, sectes. (Ib.)

Ils s'ordonnent sous des petites communautés sociales qui respectent la ritualisation de la vie quotidienne, les phantasmes et les productions de la mythologie qui dominent l'imaginaire collectif.

Donc, la pensée de la prose de Nedelciu est dialogique non seulement dans le sens de la co-participation du lecteur aux jeux de l'ironie historique-littéraire, mais aussi dans le sens de la participation du personnage à la vie pratique, à la petite histoire de l'entreprise ou de la collectivité humaine dont il est le représentant, le membre, le chef, l'élu ou l'exclu. La motivation de cette collaboration est gouvernée par une conscience terrifiée, toujours ressentie comme coupable, qui vit par ses hypothétiques états et degrés d'écart social, qui s'effraye de l'accusation, de l'exclusion, de la réjection, de la condamnation. Par exemple, la conscience condamnée à la peur et perpétuée par la terreur du régime fait que l'héros de la prose L'Effet de l'écho contrôlé, Grig, soit en garde, qu'il vérifie que sa délation ne soit pas incriminatoire, qu'elle n'incrimine ni luimême, ni son partenaire de la soirée. Dans ce contexte d'inquiétude, Grig hésite (écrire ou non?, comment raconter l'événement?, devenir un délateur ou garder son intégrité morale?) et son oscillation devient pour l'auteur un prétexte de faire une prose à final ouverte (aux interprétations, aux suspicions de la censure) qui a pu thématiser implicitement l'ambiguïté de l'écriture, la dualité de la pensé humaine, finalement le style particulier, authentique et original de cette littérature. Nous pouvons dire que l'existence des personnages de Mircea Nedelciu est réglée par une raison sociale, pragmatique, parce que leur réalité se fonde sur des comportements inquiétants, qui rélèvent une «somatographie » d'un corps morcelé et d'une conscience schizoïde, traduits comme une sociographie culturelle et artistique réalisée par une vivisection du réel et de la réalité historique. 
Une autre pensée édifie le roman de Matei Călinescu, Viaţa şi opiniile lui Zacharias Lichter / La Vie et les opinions de Zacharias Lichter, organisé sur la singularité du personnage, sur la diction solitaire de l'élection spirituelle. C'est une pensée monologique, d'un message hermétique, ésotérique, orphique. Le personnage, le mendiant fou Zacharias Lichter, symbolise la conscience morosophique (moros + sophia), non pratique, du soliloque prophétique, sans récepteur. Son existence individuelle a comme but de remettre en question toutes les grandes notions fondamentales pour l'humanité, toutes les métarécits du monde moderne (la Liberté, la Vérité, la Pensée, le Mensonge, etc.).Le discours fébrile, paralogique et translogique, fondé par le scintillement de la pensée poétique montre une conscience dilemmatique, agonisante. Il est normal que dans cette situation l'individuation soit bouleversée, l'instance sociale de l'individu soit abolie et reconnue comme une autre, a-sociale, antisociale et trans-individuelle. La réaction par le discours paralogique, la folie mimée sont les subterfuges de la liberté, les chemins cachés de l'autonomie de penser, les voiles de la solitude pleine de sens de la révolte.

La pensée du corporel, de l'intelligence de la chair, résume la poétique romanesque du prosateur Gheorghe Crăciun, dans son illustration magistrale de Pupa Russa, car ses efforts son rassemblés pour exprimer l'autonomie et la véracité des sensations, la vérité élémentaire, les données immédiates de l'existence, comme dans le syntagme bergsonien. L'histoire n'est plus occultée, au contraire, elle fait le personnage, Leontina. Cette femme ne supprime pas l'histoire, ne choisit pas des formes d'évasion, elle en profite pour se transformer, pour devenir un des ses pouvoirs, une des plus brûlantes énergies de l'implacable mécanisme socio-politique. Elle décide d'être la raison $d u$ mécanisme idéologique, qui, finalement, la dévore. L'obsession de la corporalité, de l'authenticité se trouve dans le roman exaltée dans la pluralité des codes: dénotatifs, ironiques, métaphoriques, théoriques, autoréférentiels, philosophiques, ludiques, tragiques. La philosophie du roman de Crăciun est le dévoilement progressif, comme dans le principe de matriochka, en révélant l'essence dramatique de l'être humain.

En conclusion, nous pouvons dire que l'efficacité des structures narratives de la prose roumaine des années ' 80 dépend de leur flexibilité, de leur potentiel combinatoire, générateur de formes variables, apte à produire des effets inattendus de nouveauté, d'ingéniosité, de surprise, malgré le contexte des directives de l'idéologie marxiste. Pour Mircea Nedelciu, et la chose reste valable pour toute sa génération, la littérature n'a pas de sens en l'absence d'une forte dimension créative et expérimentale. Par contradiction, les blocages 
esthétiques, politiques ont eu la fonction de mobiliser la créativité, de stimuler la liberté de la pensée, malgré les doctrines de Parti, malgré la domination de l'esprit. «Expérimenter en littérature (affirme Nedelciu) signifie écrire en tenant compte de certaines contraintes connues, auto-imposées ou imposées. L'écrivain digne de ce nom a besoin de contraintes pour que son esprit jaillisse, par l'écrit, vers la liberté. "Les formes fixes", par exemple, accomplissaient cette fonction dans la littérature classique ». (Dacă vorbim strict de ştiința de a scrie, interview de Petre Cimpoieşu, dans la revue Ateneu, apud. Lefter 2010 : 19).

Un autre témoignage paru dans la revue Tribuna, nr. 5 (1571) / 1987, prouve la cohérence de sa vision artistique:

«Je pense être une nature expérimentatrice. Je veux dire que j'évite à tout prix de produire selon une formule qui semble marcher. Je pense que ce continuel essai fait partie de l'essence de la littérature et je pense aussi que l'expériment est un devoir de la littérature si elle est une boîte de résonnance pour les confrontations d'idées du monde contemporain. L'accélération du rythme des transformations de notre actualité rend l'expérimentalisme de la littérature d'aujourd'hui plus évident. Pour moi, chaque livre n'est que le fragment d'un dialogue avec le monde». (Apud. Lefter 2010: 21).

Le militantisme de la littérature officielle qui entretenait l'esthétisation du politique dans le fascisme et le communisme, ainsi que les formules subversives et dissidentes, peut-être pas également combatives, mais marquées à leur tour par un militantisme malgré lui, un militantisme au sens différent, faux au fait, renversé, devrait être situés en contiguité, en complémentarité et opposition au désenchantement politique et idéologique, parce que l'érosion de l'idéologie intervient et elle est continuée par la dé-légitimation et la dés-esthétisation du politique. Ces processus se sont instaurés en silence, par des confusions voulues de registres, de vocabulaire à usage antiphrastique, euphémistico-ironique.

En analysant les expériments de la décennie 1980-1990, Gh. Crăciun signale le fait que c'est à ce point précis que l'on peut trouver l'ambiguïté du statut de l'expériment, dans «la crainte d'une radicalité trop saillante, le soin de la noyer dans des contextes qui amoindrissent sa force de frappe», mais qui arrivent à fonctionner en toute innocence - une innocence apparente, comme un «système de tributs indirects» (1998: 39). Ces compromissions littéraires entre écrivains, éditeurs, rédacteurs de revues, critiques et le public sont ceux qui expriment au fait la complexité des moyens de légitimation dans n'importe quelle culture située dans un régime totalitaire. 
«La syntaxe de la liberté de dire», comme l'a appelée Gheorghe Iova, devait être inventée et réinventée, puisque le sentiment de l'impuissance, de la culpabilité, de la compromission indirecte, par le silence, planait dans les consciences des écrivains qui n'ont pas flirté avec la non-valeur que pour se faire acceptés par le système éditorial. En illustrant les traits des quelques-unes des stratégies discursives de la prose roumaine des années 70-80, tel le roman essayistique de Matei Călinescu ( $L a$ Vie et les opinions de Zacharias Lichter), les proses courtes, expérimentales et les romans signées par Mircea Nedelciu et Gh. Crăciun, on a vu que ces formes apparemment esthétisantes s'opposaient au discours officiel, idéologisant du pouvoir. Au fait, elles sont l'expression d'une légitimation marquée par l'angoisse, par l'exercice fragile de la liberté d'expression de l'époque. La sourde ambiguité conflictuelle, les formulations antiphrastiques, euphémistiques, l'esthétique de l'expérimente littéraire par la recherche des jeux ironico-parodiques ont mené à l'élaboration d'une poétique transgressive qui a permis de sauver les oeuvres de la censure communiste.

\section{Bibliographie}

Bloom, H. 1994. Canonul occidental. Cărțile şi şcoala epocilor. (The Western Cann - The Books and School of the Ages.) Bucureşti: Univers, trad. D. Stanciu.

Bodiu, A. 2000. Direcția optzeci în poezia română, I. Piteşti: Ed. Paralela 45.

Bourdieu, P. 1992. Les règles de l'art. Paris: Editions du Seuil; 1998. Regulile artei. Bucureşti: Univers, trad.B. Ghiu şi T. Saulea.

Călinescu, M. 1995. Viața şi opiniile lui Zacharias Lichter. Iaşi: Polirom, III ${ }^{\mathrm{ème}}$ édition.

Crăciun, Gh. 1998. Experimentele unui deceniu - 1980-1990. - Experimentul literar românesc postbelic. Piteşti: Ed. Paralela 45.

Crăciun, Gh. 2009. Pactul somatografic, ed. îngrijită şi prefaţată de I.B. Lefter. Piteşti: Editura Paralela 45, « Mircea Nedelciu şi Curtea interioară a literaturii. Corpuri netransparente, sociografii narative, tratamente ambulatorii $\gg$.

Crăciun, Gh. 2004. Pupa Russa. Piteşti: Ed.Paralela 45.

Hamon, Ph. 1996. L'Ironie littéraire. Essais sur les formes de l'écriture oblique. Paris: Hachette, Supérieur.

Kundera, M. 2005. Le Rideau, Paris: Éditions Gallimard; 2008. Cortina: eseu în şapte părţi. Bucureşti: Humanitas, trad. Vlad Russo.

Lefter, I. B. 1998. Literatura anilor '60-'70: Experimentul ca despărțire de neo-modernism (schiță tipologică şi scurt inventar). - Experimentul literar românesc postbelic. Piteşti: Ed. Paralela 45.

Lefter, I.B. 2010. 7 postmoderni: Nedelciu, Crăciun, Müller, Petculescu, Gogea, Danilov, Ghiu. Piteşti: Ed. Paralela 45.

Macrea Toma, I. 15.06.2011. La censure institutionnalisée et incorporée. - Asymetria. Revista de cultura, critica si imaginatie, http: / / www.asymetria.org / . 
Quelques Livres du Canon Littéraire Roumain - face au pouvoir politique

http: / / www.asymetria.org / / modules.php?name=News\&file=article\&sid=333

Muşat, C. 1998. Perspective asupra romanului românesc postmodern şi alte ficțiuni teoretice. Piteşti: Ed. Paralela 45.

Nedelciu, M. 1999. Aventuri într-o curte interioară, anthologie d'auteur avec un préface de I.B. Lefter et une postface de S. Alexandrescu, Piteşti: Ed. Paralela 45.

Ricoeur, P. 2004. Sur la traduction. Paris: Bayard; 2005. Despre traducere. Iaşi: Polirom, trad. Magda Jeanrenaud.

Simuț, I. 1993. Care patru literaturi? - România literară, nr 29, 28.07-03.08.

Spiridon, M. 1998. Experimentalismul bine temperat. - Experimentul literar românesc postbelic. Piteşti: Ed. Paralela 45.

Steiner, G. 2006. Una certa idea di Europa. Milano: Garzanti Libri, prefazione di Mario Vargas Llosa.

This paper is supported by UEFISCDI, contract number 863/ 19.01.2009 and also by the Sectorial Operational Programme Human Resources Development (SOP HRD), financed from the European Social Fund and by the Romanian Government under the contract number SOP HRD/89/1.5/S/59758. 